\title{
Electroestimulación neuromuscular intradiálisis, fuerza muscular, capacidad funcional y composición corporal
}

\author{
Sandra Rubio Páez, Vicent Esteve Simó, Anna Junqué Jiménez, Ester Tomás Bernabéu, Oscar Paz López, \\ Gorka Iza Pinedo, María Luisa Lavado Sempere, Manel Ramírez de Arellano
}

Servicio de Nefrología. Hospital de Terrassa. Consorci Sanitari de Terrassa. Barcelona. España

\begin{abstract}
Resumen
Introducción: La capacidad funcional disminuida y la importante atrofia muscular caracterizan a los pacientes en hemodiálisis (HD). El ejercicio físico intradiálisis y recientemente la electroestimulación neuromuscular (EMS), representan dos serias opciones terapéuticas para mejorar esta deteriorada condición física. Actualmente, no existen estudios publicados sobre el papel de la EMS y la composición corporal en los pacientes en HD.
\end{abstract}

Objetivo: Analizar que efecto produce un programa de EMS sobre la fuerza muscular, capacidad funcional, parámetros nutricionales y composición corporal en nuestros pacientes en HD.

Material y Métodos: Estudio unicéntrico, prospectivo de 12 semanas de duración. Los pacientes incluidos realizaron un programa adaptativo de EMS en ambos cuádriceps intradiálisis mediante el dispositivo Compex R Theta 500i. Analizamos: 1.- Parámetros nutricionales (Albumina, pre albúmina, triglicéridos, colesterol total y fracciones, ferritina y Proteína C reactiva). 2.- Datos musculares: Composición muscular cuadriceps, Fuerza extensión máxima cuádriceps (FEMQ) y handgrip (HG) brazo dominante. 3.- Test funcionales: "Sit to stand to sit" (STS10) y "six- minutes walking test" (6MWT). 4.- Composición corporal mediante biompedancia electrica (BIA).

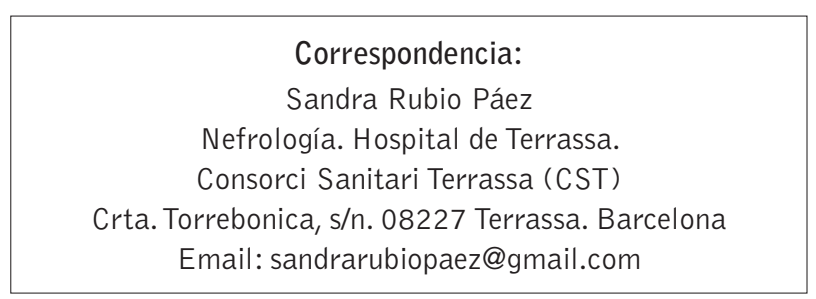

Resultados: 13 pacientes incluidos: $(69.2 \%$ hombres). Edad media: 65.7 años y 33.9 meses en HD. I.Charlson medio 9.1. La principal etiología de la ERC fue la DM ( $38.5 \%)$. Al final del estudio se observó una mejoría en ( $\left.{ }^{*} p<0.05\right)$ : FEMQ* $(11.7 \pm 7.1$ vs $13.4 \pm 7.4 \mathrm{Kg}), \operatorname{STS10}(39.3 \pm 15.5$ vs $35.8 \pm 13.7 \mathrm{seg})$, $6 \mathrm{MWT}^{*}(9.9 \%, 293.2$ vs $325.2 \mathrm{~m})$. En relación a la composición corporal, se observó únicamente un aumento significativo del área muscular (AMQ*: 128.6 \pm 30.2 vs $144.6 \pm 22.4 \mathrm{~cm}^{2}$ ) y una disminución del área grasa (AGQ*: $76.5 \pm 26.9$ vs $62.1 \pm 20.1 \mathrm{~cm}^{2}$ ) a nivel quadricipital, sin cambios en el resto de datos analizados (\% grasa abdominal, peso graso, peso magro, agua corporal total). No se objetivaron cambios relevantes en los parámetros nutricionales y de adecuación dialítica.

Conclusiones: 1.- La electroestimulación neuromuscular intradialísis mejoró la fuerza muscular, la capacidad funcional y la composición muscular del cuadriceps de nuestros pacientes en HD. 2.- Nuestros resultados remarcan el carácter local de la electroestimulación neuromuscular, dada la ausencia de cambios relevantes en el resto de los parámetros nutricionales y datos corporales analizados. 3.- No obstante, son necesarios futuros estudios mejor diseñados, de cara a discernir si la electroestimulación neuromuscular podría ser una nueva alternativa terapéutica para evitar la atrofia muscular y el deterioro progresivo de la condición física de éstos pacientes.

PALABRAS CLAVE

- ELECTROESTIMULACIÓN NEUROMUSCULAR

- HEMODIÁLISIS

- FUERZA MUSCULAR

- COMPOSICIÓN CORPORAL 


\section{Intradialysis neuromuscular electro stimulation, muscle strength, functional capacity and body composition}

\section{Abstract}

Background: The reduced functional capacity and significant muscle atrophy characterized patients on hemodialysis. Intradialytic exercise and recently neuromuscular electrostimulation (EMS) represent two serious therapeutical options to improve the deteriorated physical condition. Until date, there are no published studies about the role of EMS and body composition in HD patients.

Objectives: Analyze the effect a program of EMS on muscle strength, functional capacity, nutritional parameters and body composition in our HD patients.

Methods: A 12 weeks single-center, prospective study. Patients included in the study performed an intradialysis EMS adaptive program in both quadriceps using the Compex R Theta 500i device. We analyzed: 1.- Nutritional parameters (albumin, pre-albumin, triglycerides, total cholesterol and fractions, ferritin and C-reactive protein). 2.- Muscular data: Muscular composition, Maximum length quadriceps strength (MLQS) and "hand-grip" (HG) dominant arm. 3.- Functional capacity test: "Sit to stand to sit" (STS10) and "six- minutes walking test" (6MWT). 4.- Body composition.

Results: 13 HD patients included: $69.2 \%$ men. Mean age 65.7 years and 33.9 months on HD. A significant $\left({ }^{*} p\right.$ $<0,05)$ improvement was observed in MLQS* $(11.7 \pm 7.1$ vs $13.4 \pm 7.4 \mathrm{Kg}), \mathrm{STS} 10 *(39.3 \pm 15.5$ vs $35.8 \pm 13.7 \mathrm{seg})$, $6 \mathrm{MWT}^{*}(9.9 \%, 293.2$ vs $325.2 \mathrm{~m})$. There was a significant increase in the quadriceps muscular area (QMA*: $128.6 \pm 30.2$ vs $144.6 \pm 22.4 \mathrm{~cm}^{2}$ ) and decrease of fat quadricipital area (FQA*: $76.5 \pm 26.9$ vs $62.1 \pm 20.1$ $\mathrm{cm}^{2}$ ). No significant changes were observed in nutritional parameters, body composition (body fat percentage, lean and fat mass, total body water) or dialysis adecuacy data.

Conclusions: 1.- Intradialysis quadriceps EMS improved muscle strength, functional capacity and the quadriceps muscle composition in our HD patients. 2.- Our results underline the local aspects on EMS, given the absence of relevant changes on nutritional parameters and body composition. 3.- Future studies are manadatory in order to establish if EMS could be a new alternative to prevent muscle atrophy and the progressive deterioration of the physical condition of these patients.

\section{KEYWORDS}

\section{- NEUROMUSCULAR ELECTRO STIMULATION}

- HEMODIALYSIS

- MUSCULAR STRENGTH

- BODY COMPOSITION

\section{Introducción}

La elevada comorbilidad cardiovascular, la malnutrición, la anemia e inflamación crónica, el sedentarismo asociado a hemodiálisis (HD), así como las propias alteraciones del metabolismo de la urea podrían ser, entre otros, algunos de los diversos factores que conducirán a lo largo de su permanencia en HD a una marcada debilidad muscular e impotencia funcional ${ }^{1,2}$.

Las alteraciones metabólicas de la urea conllevan principalmente a la afectación sobretodo de las fibras musculares y de las terminaciones nerviosas del tejido músculo esquelético, que nos conducirá a la larga a una importante atrofia muscular y a la aparición de fatiga, debilidad, calambres, rampas o mioclonias 3,4 .

Por estos motivos, uno de los aspectos fundamentales en el cuidado del paciente renal debería ser proporcionar una adecuada rehabilitación física de cara a preservar la capacidad funcional y la autonomía del paciente renal ${ }^{5,6}$.

En las últimas décadas, han sido publicados diversos estudios que reportan efectos beneficiosos tras la realización de ejercicio físico durante las sesiones de HD a nivel de capacidad funcional, psicológico y de calidad de $v^{2} \mathrm{da}^{2,7}$.

En determinadas ocasiones, por las características y la gran comorbilidad asociada, algunos pacientes son incapaces de llevar a cabo estos programas de ejercicio físico en HD, no pudiendo ser realizados de forma segura y satisfactoria. En este sentido, recientemente cobra gran interés el papel de la electroestimulación neuromuscular (EMS) como tratamiento coadyuvante del ejercicio físico regular en pacientes en $\mathrm{HD}^{8,9}$. La EMS consiste en la estimulación de grupos musculares mediante corrientes eléctricas de baja intensidad a través de unos electrodos aplicados sobre la superficie corporal. Estos impulsos estimulan los nervios con el fin de enviar señales a un músculo, el cual reacciona contrayéndose, igual que haría con la actividad muscular normal'10,11. En la población sana, su uso está ampliamente extendido en la mejora de la condición física y fuerza muscular en personas con ac- 
tividad física o deportiva. También están destinadas en la rehabilitación de grupos musculares principalmente en poblaciones con graves trastornos motores neurológicos o traumatológicos ${ }^{12,13}$.

No obstante, todavía no se dispone de suficiente evidencia acerca del papel exclusivo de la EMS sobre algunos aspectos tan importantes en la prevención del deterioro funcional progresivo como la fuerza o composición muscular en los pacientes en HD. En nuestro estudio pretendemos analizar el efecto de un programa exclusivo de EMS sobre la fuerza muscular, capacidad funcional y composición corporal en nuestros pacientes en HD.

\section{Material y Métodos}

Entre los meses de septiembre a noviembre de 2013 se realizó un estudio unicéntrico prospectivo de 12 semanas de duración aprobado por el Comité Ético de nuestra Institución y realizado de acuerdo con las normas de la declaración de Helsinki para observar el efecto de un programa exclusivo de EMS sobre la fuerza muscular, la capacidad funcional y la composición corporal de nuestros pacientes en HD.

El programa de HD periódica de nuestro hospital, distribuye a los pacientes principalmente en 6 grupos de 10 12 pacientes. Éstos grupos realizan sesiones de $4 \mathrm{~h}$ en dias alternos (L-X-V ó M-J-S), en unos horarios de mañana, mediodia y tarde. El programa exclusivo de EMS y la valoración de la composición corporal fue realizado por nuestro personal de enfermería, ya que no se disponía de recursos específicos destinados para ello.

Aquellos pacientes de los turnos de mañana y tarde que cumplían los criterios de inclusión preestablecidos, constituyeron el grupo electro estimulación (EM). Los pacientes continuaron con su actividad física diaria de forma habitual, sin prescribir ningún programa de ejercicio físico de forma adicional.

Como criterios de inclusión se establecieron: otorgar el consentimiento informado, tener una edad igual o superior a 18 años, permanencia en HD superior a 3 meses en nuestro centro y estabilidad clínica y hemodinámica en los últimos 3 meses.

Los criterios de exclusión establecidos fueron: presencia de evento cardiovascular reciente, presencia de acceso vascular interno para HD en extremidades inferiores, ser portador de marcapasos, imposibilidad física en extremidades inferiores para realizar el análisis de composición corporal y no otorgar el consentimiento informado por escrito.

\section{Electro estimulación Neuromuscular}

Los pacientes incluidos realizaron un programa de EMS de los músculos cuádriceps de ambas extremidades inferiores previamente consensuado con el servicio de rehabilitación de nuestro hospital. El dispositivo utilizado era el modelo Compex ${ }^{\circledR}$ Rehab Theta 500i, dotado de diversos programas de ejercicio rehabilitador con distintas fases, tipos e intensidad de corriente. El programa de electro estimulación incluía un programa de tonificación en la primera semana, 1 semana de resistencia - aeróbica, 2 semanas de rehabilitación - amiotrofia, 2 semanas de rehabilitación - hipertrofia, 3 semanas de potenciación muscular y finalmente 3 semanas de fuerza - resistencia. Se realizaba durante las primeras dos horas de cada sesión de HD, con una duración media de 30-45 min. Los pacientes se encontraban en su posición habitual de HD en decúbito supino, con extensión completa de los miembros inferiores y mínima flexión $\left(15^{\circ}\right)$ de ambas rodillas mediante una almohada blanda colocada en la región poplítea de las mismas. Cada paciente tenía siempre sus propios electrodos $(5 \times 10 \mathrm{~cm})$. Éstos se colocaban de forma precisa sobre el punto motor de los vientres musculares del cuádriceps (recto anterior, vaso interno y externo), garantizando la máxima comodidad y eficiencia del programa.

Coincidiendo con las visitas médicas trimestrales de seguimiento habitual programadas los días de no HD en nuestros pacientes se analizaron las siguientes variables tanto al inicio como al final del estudio.

\section{Datos demográficos, bioquímicos, parámetros nutri- cionales y antropométricos}

Las variables demográficas incluían la edad, el sexo, la etiología renal, el índice de comorbilidad de Charlson y tiempo de permanencia en HD. Del mismo modo se recogieron los principales datos bioquímicos en $\mathrm{HD}$, parámetros nutricionales (albúmina, pre-albúmina, triglicéridos, colesterol total y sus fracciones, ferritina) y de adecuación de HD (Kt/v método $2^{\mathrm{a}}$ generación Daugirdas).

El área total (ATC), muscular (AMC) y grasa (AGC) de ambos cuadriceps se obtuvieron mediante las fórmulas antropométricas de Gurney y Jelliffe ${ }^{14}$ : Área total (ATQ) $=[\text { Contorno músculo }(\mathrm{cm})]^{2} / 4 \pi$. Área Muscular (AMQ) $=[$ (Contorno músculo $(\mathrm{cm})-\pi \times$ Pliegue cutáneo músculo $\left.(\mathrm{cm})^{2}\right] / 4 \pi$. Área Grasa $(A G C)=$ Área total $(A T C)$ - Área Muscular (AMC). El contorno muscular se estimó en su posición anatómica de referencia mediante centimetría, con una cinta flexible e inextensible y expresada 
en centímetros sin comprimir los tejidos blandos de la zona. El pliegue cutáneo de ambos cuádriceps se utilizó para la valoración del tejido adiposo subcutáneo. Mediante un plicómetro, estimamos el espesor del pliegue de la piel, es decir una doble capa de piel y tejido adiposo subyacente, evitando siempre incluir el músculo en el punto medio longitudinal de la línea que une el pliegue inguinal y borde proximal de la rótula, en la cara anterior del muslo, con el paciente apoyando los pies en el suelo y formando sus rodillas un ángulo de $90^{\circ}{ }^{15}$.

\section{Fuerza muscular y capacidad funcional}

Para la valoración de la fuerza muscular de las extremidades superiores se utilizó un dinamómetro homologado tipo Jamar (Hand-grip dynamometer) (HG) en el brazo dominante (SH 5001, Seahan Corporation, Korea). Se realizó con el sujeto en pie, con los brazos extendidos a lo largo del cuerpo y se le entregó el dinamómetro en ambos brazos indicándole que hiciera la mayor fuerza posible sin apoyar el brazo en el cuerpo. El brazo que presentó una mayor fuerza, fue considerado como brazo dominante ${ }^{16}$.

Para la valoración de la fuerza muscular en EEII se utilizó un dinamómetro de tracción homologado tipo Kern (Kern CH50 50KG dynamometer). Se estimó la fuerza máxima de extensión de los músculos cuádriceps (FEMQ) de la pierna izquierda. El paciente permanecía sentado en una silla fija de tal forma que la espalda quedaba apoyada en el respaldo y la cadera y la rodilla a $90^{\circ}$. En esta posición se colocaba una cincha de sujeción inextensible a la altura del tercio distal de la tibia y se le pedía al sujeto que hiciera la mayor fuerza posible para realizar la extensión de la extremidad sin agarrarse con los brazos a la silla ${ }^{17}$.

Los resultados obtenidos tanto en las variables antropométricas, como de fuerza muscular, representan la media de tres medidas consecutivas y fueron realizadas por el mismo profesional a fin de evitar posibles errores de medición.

Las pruebas utilizadas para la valoración de la capacidad funcional fueron el test de los 6 minutos de la marcha (6MWT) y el test STS10 (sit to stand to sit 10). El test 6MWT se realizó con monitorización de las constantes habituales y la saturación de oxigeno mediante pulsioximetría. Consistía en evaluar la máxima distancia recorrida durante un período de 6 minutos a ritmo activo, a lo largo de un pasillo de 20 metros cercano a la unidad de hemodiálisis. Transcurrido el tiempo de la prueba se registraba la distancia total recorrida mediante un odómetro homologado18. EI Test STS 10 consistía en levantarse y volverse a sentar durante 10 veces consecutivas lo más rápido posible; partiendo de una posición sentada con los brazos pegados al pecho desde una silla de $44.5 \mathrm{~cm}$ de alto y $38 \mathrm{~cm}$ de profundidad apoyada contra la pared para evitar el riesgo de caídas. Se anotaba el tiempo en segundos que se tardaba en realizar el ejercicio ${ }^{19}$.

\section{Análisis de la composición corporal (bioimpedancia eléctrica)}

El análisis de la composición corporal se realizó mediante bioimpedancia eléctrica utilizando el dispositivo homologado OMRON BF-400 (Omron Healthcare UK LTD, Japón). Se realizó con el sujeto descalzo, en apoyo uniforme en bipedestación y completamente estático, con ambos brazos pegados a ambas partes del cuerpo sobre la plataforma del dispositivo a los 15 minutos de finalizar la tercera sesión semanal de hemodiálisis. Una segunda determinación se repitió, en las mismas condiciones, a los 5 minutos de la inicial, obteniendo el valor promedio. Se analizaron los siguientes parámetros: peso total (PT), peso graso $(P G)$, peso magro $(P M)$, agua corporal total (ACT) y porcentaje de grasa abdominal (\%GA). EI PG y PM se estimaron mediante la fórmula de Hume ${ }^{20}: P G=[$ Peso total $\left.(\mathrm{kg}) \times \% G A)\right] / 100 . P M=$ Peso total $(\mathrm{kg})$ - Peso graso. El ACT se calculó mediante la fórmula estimada de Watson ${ }^{21}$.

El análisis estadístico se realizó con el programa SPSS versión 19.0 (SPSS Inc, Chicago, IL, EE. UU.). Las variables cuantitativas se expresaron mediante la media y desviación estándar. Las variables cualitativas, mediante porcentaje. La comparación de los datos cuantitativos al finalizar el estudio se realizó mediante el test de Wilcoxon para variables relacionadas no paramétricas y los datos cualitativos mediante el test de McNemar, considerando significación estadística aquellas relaciones con un valor de $p \leq 0,05$.

\section{Resultados}

Un total de 13 pacientes superaron los criterios de inclusión y fueron analizados. Ninguno de los pacientes presentó efectos adversos relacionados con la EMS. EI $69.2 \%$ eran hombres, con una edad media de $65.7 \pm$ 12.8 y un tiempo de permanencia en HD de $33.9 \pm 24.7$ meses. El índice medio de Charlson fue de $9.1 \pm 2.3$. Las principales etiologías de la insuficiencia renal de nuestros pacientes fueron la diabetes mellitus (38.5\%), la pielonefritis crónica o de repetición (23.1\%), enfermedad glomerular (15.4\%), y HTA (7.7\%) enfermedad no filiada $(7.7 \%)$ y otras $(7.7 \%)$. 
Los resultados relativos a la valoración de la fuerza muscular y los datos relativos a la capacidad funcional se muestran en la tabla 1.

Tabla 1. Valoración de la fuerza muscular y la capacidad funcional. Inicio vs Final del estudio. HG: Hand Grip brazo dominante. FEMQ: Fuerza Extensión máxima del cuádriceps.. 6MWT: Test de la marcha 6 min. STS10: test sit to stant to sit 10;m: metros; seg: segundos. Significación estadística: $p<0,05$ ).

\begin{tabular}{|l|c|c|c|}
\hline & Inicio & Final & Sig. est \\
\hline HG (kg) & $21.2 \pm 9.3$ & $21.6 \pm 9.8$ & 0.616 \\
\hline FEMQ (kg) & $11.7 \pm 7.1$ & $13.4 \pm 7.4$ & $0.002^{*}$ \\
\hline 6MWT (m) & $293.2 \pm 163.9$ & $325.2 \pm 176.4$ & $0.018^{*}$ \\
\hline STS10 (sec) & $39.3 \pm 15.5$ & $35.8 \pm 13.7$ & 0.310 \\
\hline
\end{tabular}

No obtuvimos cambios significativos en la valoración de la fuerza muscular mediante el $\mathrm{HG}$ al finalizar el estudio. Por el contrario, sí que observamos una mejoría significativa de la $F M E Q$ de las extremidades inferiores (FMEQ $11.7 \pm 7.1$ vs $13.4 \pm 7.4 \mathrm{~kg} ; p 0.002$ ). En relación al test $6 \mathrm{MWT}$, observamos un incremento significativo del $9.9 \%$ en la distancia recorrida al finalizar el estudio ( $293.2 \pm 163.9$ vs $325.2 \pm 176.4 \mathrm{~m}$, p 0.018). En el test STS10 se observó un menor tiempo en la realización del mismo $(39.3 \pm 15.5$ vs $35.8 \pm 13.7$ seg, $p$ 0.310 ) al finalizar el estudio, si bien estas diferencias no alcanzaron la significación estadística preestablecida.

La tabla 2 representa los datos obtenidos en relación a la composición muscular del cuádriceps. Únicamente obtuvimos un incremento significativo del valor promedio del AMC (128.6 \pm 30.3 vs $144.6 \pm 22.4 \mathrm{~cm}^{2}, p$ 0.032) así como una disminución significativa del valor promedio de AGC $\left(76.5 \pm 26.9\right.$ vs $\left.62.1 \pm 20.1 \mathrm{~cm}^{2}, p 0.024\right)$ al final del estudio. No se observaron cambios relevantes en relación al valor promedio del ATC.
Tabla 2. Valoración de la fuerza muscular. Inicio vs Final del estudio. ATQ. Área total del cuadriceps. AGQ. Área grasa del cuádriceps. AMQ. Área muscular del cuádriceps. Significación estadística: $\left.{ }^{*} p<0,05\right)$.

\begin{tabular}{|c|c|c|c|}
\hline & Inicio & Final & Sig. est \\
\hline \multicolumn{4}{|l|}{$\begin{array}{l}\text { Área Total } \\
\text { Cuadriceps }\left(\mathrm{cm}^{2}\right)\end{array}$} \\
\hline Cuadriceps D & $205.6 \pm 38.3$ & $197.1 \pm 33.8$ & 0.221 \\
\hline Cuadriceps I & $204.8 \pm 41.3$ & $204.3 \pm 37.1$ & 0.932 \\
\hline Promedio ATC & $205.2 \pm 39.1$ & $200.7 \pm 34.7$ & 0.472 \\
\hline \multicolumn{4}{|l|}{$\begin{array}{l}\text { Área Muscular } \\
\text { Cuadriceps }\left(\mathrm{cm}^{2}\right)\end{array}$} \\
\hline Cuadriceps D & $129.2 \pm 28.9$ & $142.2 \pm 19.8$ & 0.166 \\
\hline Cuadriceps I & $128.1 \pm 33.7$ & $146.1 \pm 28.6$ & $0.029 *$ \\
\hline Promedio AMC & $128.6 \pm 30.3$ & $144.6 \pm 22.4$ & $0.032 *$ \\
\hline \multicolumn{4}{|l|}{$\begin{array}{l}\text { Área Grasa } \\
\text { Cuadriceps }\left(\mathrm{cm}^{2}\right)\end{array}$} \\
\hline Cuadriceps D & $76.2 \pm 26.2$ & $59.9 \pm 21.2$ & $0.005^{*}$ \\
\hline Cuadriceps I & $76.7 \pm 29.1$ & $64.2 \pm 20.4$ & 0.108 \\
\hline Promedio AGC & $76.5 \pm 26.9$ & $62.1 \pm 20.1$ & $0.024 *$ \\
\hline
\end{tabular}

En relación a la composición corporal estimada mediante bioimpedancia eléctrica no se encontraron cambios relevantes. Un ligero incremento del peso magro $(56.2$ \pm 10.9 vs $57.8 \mathrm{~kg} \pm 11.6, p 0.247$ ) así como una cierta disminución del peso graso ( $15.4 \pm 7.8$ vs $13.9 \mathrm{~kg} \pm 7.7$, p 0.278), del porcentaje de grasa abdominal ( $21.3 \%$ vs $19.5 \%, p 0.262)$ y de agua corporal total $(36.4 \pm 4.9$ vs $35.5 \pm 6.3$ litros, $p$ 0.534) se observaron al final del estudio, si bien todos estos resultados no alcanzaron la significación estadística preestablecida (tabla 3).

Tabla 3. Valoración de la composición corporal mediante bioimpedancia eléctrica (BIA). Inicio vs Final del estudio. ACT: Agua corporal total. L: litros. Kg: kilogramos. Significación estadística: ${ }^{*} \mathrm{p}<0.05$.

\begin{tabular}{|l|c|c|l|}
\hline & Inicio & Final & Sig. est \\
\hline ACT (L) & $36.4 \pm 4.9$ & $35.5 \pm 6.3$ & 0.534 \\
\hline Peso total $(\mathbf{k g})$ & $71.6 \pm 9.8$ & $71.7 \pm 10.5$ & 0.817 \\
\hline Peso graso(kg) & $15.4 \pm 7.8$ & $13.9 \pm 7.7$ & 0.278 \\
\hline Peso magro(kg) & $56.2 \pm 10.9$ & $57.8 \pm 11.6$ & 0.247 \\
\hline$\%$ grasa abdominal & $21.3 \%$ & $19.5 \%$ & 0.262 \\
\hline
\end{tabular}


Los principales datos bioquímicos y parámetros de adecuación dialítica se muestran en la tabla 4.

Tabla 4. Datos bioquímicos y parámetros nutricionales. Inicio vs Final del estudio. Colest: Colesterol. Significación estadística: ${ }^{*} p<0.05$.

\begin{tabular}{|l|c|c|c|}
\hline & Inicio & Final & Sig.est \\
\hline Albúmina (g/dL) & $3.7 \pm 0,3$ & $3.7 \pm 0,2$ & 0.895 \\
\hline PreAlbúmina (mg/dl) & $28.5 \pm 10.5$ & $27.1 \pm 4.8$ & 0.636 \\
\hline Colest Total (mg/dl) & $143.2 \pm 57.2$ & $148.1 \pm 59.1$ & 0.193 \\
\hline Colest HDL (mg/dl) & $42.6 \pm 11.1$ & $41.2 \pm 11.9$ & 0.487 \\
\hline Colest LDL (mg/dl) & $68.7 \pm 42.3$ & $63.4 \pm 49.2$ & 0.482 \\
\hline Triglicéridos (mg/dl) & $154.1 \pm 76.2$ & $158.6 \pm 73.6$ & 0.742 \\
\hline Hemoglobina (g/dl) & $10.4 \pm 1.8$ & $11.1 \pm 0.9$ & 0.276 \\
\hline Ferritina $(\mathbf{n g} / \mathrm{mL})$ & $401.6 \pm 210.7$ & $433.2 \pm 267.8$ & 0.820 \\
\hline
\end{tabular}

No se encontraron diferencias significativas al finalizar el estudio en relación a los principales parámetros bioquímicos nutricionales y principales datos de adecuación dialítica analizados (Ktv Daugirdas $2^{\mathrm{a}}$ gen: $1.63 \pm 0.4$ vs $1.62 \pm 0.7$ ). Del mismo modo, no se realizaron modificaciones en la dosis media de agentes eritropoyéticos prescrita de forma habitual en ninguno de nuestros pacientes a lo largo del estudio $(25.6 \pm 11.6$ vs $26.2 \pm$ 12.4 mcg darbepoetina/semana).

\section{Discusión}

La elevada comorbilidad cardiovascular, la malnutrición, la anemia e inflamación crónica, el sedentarismo asociado a HD, así como las propias alteraciones del metabolismo de la urea podrían ser, entre otros, algunos de los diversos factores que conducirán a lo largo de su permanencia en HD a una marcada debilidad muscular e impotencia funcional ${ }^{1,2}$.

Las alteraciones metabólicas de la urea conllevan principalmente a la afectación sobretodo de las fibras musculares y de las terminaciones nerviosas del tejido músculo esquelético, que nos conducirá a la larga a una importante atrofia muscular y a la aparición de fatiga, debilidad, calambres, rampas o mioclonias ${ }^{3,4}$.

Una de las estrategias utilizadas en la prevención y tratamiento de la pérdida muscular en estos pacientes ha sido en los últimos años, la realización de ejercicio físico en $\mathrm{HD}^{2,7}$. En este sentido, uno de los aspectos funda- mentales en el cuidado del paciente renal debería ser proporcionar una adecuada rehabilitación física de cara a preservar la capacidad funcional y la autonomía del paciente renal ${ }^{5,6}$.

Recientemente cobra gran interés el papel de la EMS como terapia coadyuvante al ejercicio físico, dado que en algunas ocasiones, por las características clínicas y la gran comorbilidad asociada los pacientes son incapaces de llevar a cabo estos programas de ejercicio físico en HD. Los escasos estudios publicados en la literatura en relación al papel de la EMS, fundamentalmente en pacientes con insuficiencia cardíaca crónica o patología pulmonar, muestran efectos favorables sobre la composición muscular y capacidad funcional8-13. Además destacan por ser fáciles de aplicar, presentar un perfil de seguridad elevado y la ausencia de graves complicaciones $^{11,12}$.

Tras una revisión exhaustiva de la literatura reciente, únicamente encontramos pequeños estudios publicados en relación a la EMS en pacientes renales. Dobsak et $\mathrm{al}^{8}$, en un estudio randomizado de 20 semanas de duración con 3 grupos comparativos, observó que tanto un programa exclusivo de EMS como un programa de EMS asociado al uso de cicloergómetros en 32 pacientes en HD, fueron capaces de mejorar la fuerza muscular, la capacidad funcional, la calidad de vida así como los parámetros de diálisis respecto a un tercer grupo control sin intervención. Del mismo modo, Farese et al ${ }^{9}$, en un estudio similar con 9 pacientes, observó un incremento de las cifras tensionales y una reducción significativa de los valores urea y fósforo tras un programa de EMS durante 9 sesiones consecutivas de HD en los grupos electro estimulados y entrenados respecto $a$ un grupo control.

A nivel nacional, merece la pena destacar los dos únicos estudios publicados acerca de la EMS asociada al ejercicio. Resultados similares a los previamente publicados fueron obtenidos por nuestro grupo de trabajo en términos de fuerza muscular, capacidad funcional y calidad de vida en 11 pacientes en HD periódica en un estudio recientemente publicado, acerca del papel de la EMS asociado al ejercicio físico de predominio aeróbico mediante el uso de cicloergómetros; aportando de forma adicional datos favorables sobre la seguridad, eficacia y tolerabilidad de la EMS en las sesiones de HD²2. Igualmente, Contreras et $\mathrm{al}^{23}$, en un grupo de 11 pacientes en HD mostraron también una mejoría en estos términos tras la realización de un programa de 5 semanas de duración fuerza-resistencia asociada a EMS de ambos cuádriceps en las sesiones de HD. 
En el presente estudio observamos una mejoría de la fuerza muscular y la capacidad funcional tras un programa exclusivo de EMS en nuestros pacientes en HD. Éstos resultados son prácticamente superponibles a los obtenidos en los estudios previamente publicados, si bien, a diferencia de éstos, nuestro estudio aporta de forma adicional algunos datos interesantes en relación a los cambios en la composición muscular tras el programa de EMS.

Ampliamente han sido descritos los múltiples efectos beneficiosos del ejercicio físico a nivel cardiovascular, psicológico o muscular. A nivel muscular, se caracterizan por un incremento de la fuerza, resistencia y tamaño de los grupos musculares ejercitados; así como por los consecuentes cambios en la composición corporal en forma de disminución de la grasa abdominal, incremento de la masa magra y tejido muscular, disminución de los pliegues cutáneos 0 incremento del diámetro muscular ${ }^{24,25}$. Estos cambios en la composición corporal han sido descritos tras el uso de la EMS de forma global ${ }^{26}$.

De todos modos, la EMS se basa en la aplicación de impulsos repetitivos de baja frecuencia mediante unos electrodos de superficie, consiguiendo la inmediata activación local y reclutamiento de fibras musculares de pequeño tamaño de los diferentes grupos musculares ${ }^{10-12}$. Precisamente esta activación y reclutamiento muscular local, podría justificar, la mejoría significativa de la fuerza muscular exclusivamente en ambos cuádriceps (FEMQ) así como la ausencia de cambios en el $H G$, un marcador de fuerza muscular en extremidades superiores y de condición física global en pacientes ancianos $^{2,6,16}$, así como la ausencia de cambios relevantes en los principales datos bioquímicos y parámetros nutricionales analizados o en la composición corporal estimada mediante bioimpedancia eléctrica. En este sentido, sería necesario electro estimular de forma global todo el organismo o bien realizar programas de electro estimulación combinados con ejercicio físico, en los que se reclutan un mayor número de grupos musculares y el efecto es más generalizado, de cara a obtener cambios relevantes en estas estimaciones bioquímicas y de composición corporal global.

A nivel de la composición muscular del cuádriceps, este evidente incremento de fuerza muscular en ambas EEII; quedó reflejado en un incremento significativo del área muscular del cuádriceps así como en una disminución significativa del área grasa muscular. La mecanismos involucrados en la aparición de estos cambios musculares estructurales son múltiples y complejos; si bien se fundamentan en el incremento del aporte de oxígeno a los tejidos o la disminución de ciertas citoquinas pro inflamatorias (IFN y, IL-6) secundaria a la electro estimulación repetitiva y continuada a nivel muscular ${ }^{27,28}$.

A pesar de estos significantes hallazgos musculares, no se observaron cambios relevantes a nivel del área total del mismo. Estos datos podrán ser atribuidos, entre otros, a la escasa duración del programa específico de hipertrofia muscular así como a la elevada atrofia muscular consecuente de la longeva permanencia en HD de nuestros pacientes, si bien tampoco podemos descartar algún error en las mediciones realizadas a pesar de la metodología descrita. Tal vez, un programa de EMS de mayor duración o quizá el uso de exploraciones complementarias no basadas en las fórmulas de estimación muscular antropométricas, como la ecografía, la tomografía computerizada o la resonancia magnética muscular; pudiesen detectar con mayor precisión cambios antropométricos musculares totales.

En relación a los test funcionales, tanto el 6MWT como el STS 10, son tests funcionales indicadores de la fuerza muscular del cuádriceps ampliamente utilizados en la práctica clínica 2,6,19. La aplicación de un programa de EMS se tradujo en un incremento significativo en la distancia recorrida en el test de la marcha. Este incremento pone de manifiesto la activación muscular de los músculos cuádriceps y el papel fundamental de la EMS en el fortalecimiento de las extremidades inferiores. Curiosamente, no obtuvimos cambios significativos en el test STS10. Estos resultados podrían atribuirse a la gran variabilidad de los resultados obtenidos en la realización del test, la dificultad en la propia realización del STS10 en éste particular tipo de pacientes en HD, así como al limitado número de pacientes.

Entre las múltiples limitaciones de nuestro trabajo, mencionar la ausencia de grupo control, el escaso tamaño de la muestra que obligó al uso de test no paramétricos así como la duración del programa de EMS, si bien son similares en cuanto número y duración a los previamente publicados. En este sentido, quedaría abierta la necesidad de realizar estudios mejor diseñados para establecer el exclusivo papel de la EMS a largo plazo y sus potenciales efectos beneficiosos en este tipo de pacientes.

En conclusión, la electro estimulación neuromuscular intradiálisis de ambos cuádriceps mejoró la fuerza muscular, la capacidad funcional y la composición muscular de nuestros pacientes en HD de una forma segura y eficaz. En espera de futuros estudios, el electro estimulación neuromuscular constituye una novedosa alternativa terapéutica para mejorar la condición física y la composición 
muscular de éstos pacientes, especialmente en aquellos pacientes en los que la realización de un programa de ejercicio físico intradiálisis sea dificultosa o esté contraindicada.

\section{Agradecimientos}

A todos los pacientes y personal de enfermería por su valiosa colaboración en la presente investigación en cuanto han hecho que este trabajo saliera adelante de la mejor manera posible.

Recibido: 10 agosto 2015

Revisado: 14 septiembre 2015

Modificado: 22 septiembre 2015

Aceptado: 2 octubre 2015

\section{Bibliografía}

1. Cheema BS, Singh MA. Exercise training in patients receiving maintenance hemodialysis: a systematic review of clinical trials. Am J Nephrol 2005; 25 (4):352-64.

2. Segura-Ortí E.Exercise in haemodyalisis patients: a literature systematic review. Nefrologia 2010; 30 (2):236-46.

3. Johansen KL, Shubert T, Doyle J, Soher B, Sakkas GK, Kent-Braun JA. Muscle atrophy in patients receiving hemodialysis: effects on muscle strength, muscle quality, and physical function. Kidney Int 2003; 63 (1):291-7.

4. Sakkas GK, Ball D, Mercer TH, Sargeant AJ, Tolfrey $K$, Naish PF. Atrophy of non-locomotor muscle in patients with end-stage renal failure. Nephrol Dial Transplant 2003; 18 (10):2074-81.

5. Painter P, Roshanravan B. The association of physical activity and physical function with clinical outcomes in adults with chronic kidney disease. Curr Opin Nephrol Hypertens 2013; 22(6):615-23.

6. Painter $P$, Marcus RL. Assessing physical function and physical activity in patients with CKD. Clin J Am Soc Nephrol 2013; 8(5):861-72.

7. Heiwe $S$, Jacobson SH. Exercise training in adults with CKD: A systematic review and meta-analysis. Am J Kidney Dis. 2014; 64(3):383-93.

8. Dobsak $P$, Homolka $P$, Svojanovsky J, Reichertova A, Soucek M, Novakova $M$, et al. Intra-dialytic electrostimulation of leg extensors may improve exercise tolerance and quality of life in hemodialysis patients. Art Organs 2012; 36 (1):71-8.

9. Farese $S$, Budmiger R, Aregger F, Bergmann I, Frey FJ, Uehlinger DE. Effect of transcutaneous electrical muscle stimulation and passive cycling movements on blood pressure and removal of urea and phosphate during hemodialysis. Am J Kidney Dis 2008; 52(4):745-52.

10. Maffiuletti NA, Zory R, Miotti D, Pellegrino MA, Jubeau M, Bottinelli R. Neuromuscular adaptations to electrostimulation resistance training. Am J Phys Med Rehabil 2006; 85 (2):167-75.

11. Miller C, Thépaut-Mathieu C. Strength training by electrostimulation conditions for efficacy: Int $\mathrm{J}$ Sports Med 1993; 14 (1):20-8.

12. VaIenti F. Neuromuscular electroestimulation in clinical practice. Acta Anaesthesiol 1964; 15:22745.

13. Sheffler LR, Chae J. Neuromuscular electrical stimulation in neurorehabilitation. Muscle Nerve 2007; 35 (5):562-90.

14. Gurney M, Jelliffe DB, Neill J. Anthropometry in the differential diagnosis of protein-calorie malnutrition. J Trop Pediatr Environ Child Health 1972; 18 (1):1-2.

15. Wang J, Thornton JC, Kolesnik S, Pierson RN Jr. Anthropometry in body composition. An overview. Ann N Y Acad Sci 2000; 904:317-26.

16. Leal V0, Mafra D, Fouque D. Use of handgrip strength in the assessment of the muscle function of chronic kidney disease patients on dialysis: a systematic review. Nephrol Dial Transplant 2011; 26 (4):1354-60.

17. Fisher NM, Pendergast DR, Calkins EC. Maximal isometric torque of knee extension as a function of muscle length in subjects of advancing age. Arch Phys Med Rehabil 1990; 71 (10):729-34. 
18. American Thoracic Society. ATS statement: Guidelines for the six-minute walk test. Am J Respi Crit Care Med 2002; 166 (1): 111-7.

19. Greenwood SA, Lindup H, Taylor K, Koufaki P, Rush $\mathrm{R}$, Macdougall IC et al. Evaluation of a pragmatic exercise rehabilitation programme in chronic kidney disease. Nephrol Dial Transplant 2012; 27 (Suppl 3):iii126-34.

20. Hume R. Prediction of lean body mass from heigth and weight. J. Clin Pathol 1966; 19(4):389-91.

21. Watson PE, Watson ID, Batt RD: Total body water volumes for adult males and females estimated from simple anthropometric measurements. Am J Clin Nutr 1980; 33(1): 27-39.

22. Junqué $A$, Esteve $V$, Iza G, Tomás $E$, Luceño I, Paz 0 et al. Resultados de un programa de ejercicio físico combinado con electroestimulación neuromuscular en pacientes en hemodiálisis. Rev Soc Enferm Nefrol 2013; 16 (3):161-8.

23. Contreras Martos G, Delgado M, Martínez Villar J, Parra I, Borrego F, Segura P. Eficacia de un programa de entrenamiento intradiálisis de fuerzaresistencia en combinación con electroestimulación neuromuscular: mejora de la capacidad funcional, fuerza y calidad de vida. Rev Soc Enferm Nefrol 2011; 14 (2):112-19.
24. Bae YH, Lee SM, Jo JI. Aerobic training during hemodialysis improves body composition, muscle function, physical performance, and quality of life in chronic kidney disease patients. J Phys Ther Sci 2015; 27(5):1445-9.

25. Gould DW, Graham-Brown MP, Watson EL, Viana $\mathrm{JL}$, Smith AC. Physiological benefits of exercise in pre-dialysis chronic kidney disease. Nephrology (Carlton) 2014; 19(9):519-27.

26. Kemmler W, von Stengel S. Whole-body electromyostimulation as a means to impact muscle mass and abdominal body fat in lean, sedentary, older female adults: subanalysis of the TEST-III trial. Clin Interv Aging. 2013; 8: 1353-64.

27. Hu L, Klein JD, Hassounah F, Cai H, Zhang C, Xu $\mathrm{P}$, Wang XH. Low-frequency electrical stimulation attenuates muscle atrophy in CKD--a potential treatment strategy. J Am Soc Nephrol. 2015; 26(3):626-35.

28. Pette D, Vrbová G. What does chronic electrical stimulation teach us about muscle plasticity? Muscle Nerve 1999; 22(6):666-77. 